\title{
Stigmatization, psychological and emotional trauma among frontline health care workers treated for COVID-19 in Lagos State, Nigeria: A qualitative study
}

AYI VANDI KWAGHE ( $\square$ hyelni_vandi@yahoo.com )

Federal Ministry of Agriculture and Rural Development https://orcid.org/0000-0002-7726-6259

Olayinka Stephen llesanmi

Department of Community Medicine, College of Medicine, University of Ibadan and University College Hospital Ibadan, Nigeria

\section{Peter Okpeh Amede}

Nigeria Field Epidemiology and Laboratory Training Programme

James Olatunde Okediran

Nigeria Field Epidemiology and Laboratory Training Programme

Rowland Utulu

Nigeria Field Epidemiology and Laboratory Training Programme

Muhammad Shakir Balogun

Nigeria Field Epidemiology and Laboratory Training Programme

\section{Research article}

Keywords: Stigmatization, psychological trauma, moral trauma, isolation, challenges, recommendations, Lagos State

Posted Date: September 9th, 2020

DOI: https://doi.org/10.21203/rs.3.rs-53453/v1

License: (c) (7) This work is licensed under a Creative Commons Attribution 4.0 International License. Read Full License

Version of Record: A version of this preprint was published at BMC Health Services Research on August 21st, 2021. See the published version at https://doi.org/10.1186/s12913-021-06835-0. 


\section{Abstract \\ Background}

COVID-19 pandemic has resulted in global health and economic crisis. We investigated the experiences of frontline health care workers recovering from COVID-19 in Lagos State Nigeria.

\section{Methods}

We conducted a qualitative study among frontline health workers recovering from COVID-19 in Lagos State, Nigeria. We interviewed 12 respondents based on data saturation. We used a checklist of questions to guide the interview according to the phenomenon under study. Data obtained was analyzed using Colaizzi's phenomenological method.

\section{Results}

The study was summarized under five themes: knowledge of COVID-19, exposure, reactions, challenges and recommendations. The respondents were quite knowledgeable on COVID-19, their reactions when informed of their status were denial, anxiety, distress, disorientation, crying for fear of stigmatization, while some were psychologically prepared. Reactions from colleagues, family and friends were encouraging and provided solace for them with a few colleagues and families that had negative reactions. Challenges include anosmia, movement restriction, loneliness, worries about the state of their families, nondisclosure of status to family members, non-conducive isolation centre with limited space, insomnia, stigmatization by health workers at the isolation centre, extended duration of stay, delay in the release of test results and use of ambulance for evacuation to the isolation centres. Coping strategies were watching movies, phone calls, use of social media, listening to music, attending webinars, working on projects and reading spiritual books. Recommendations were early laboratory testing of samples and conveying of results, increase testing capacity, the need of health care workers to be more compassionate, better method of evacuation of people that tested positive to COVID-19, aside the use of ambulance that increases the likelihood of stigmatization and standard guideline for the case management of people recovering from COVID-19 in Lagos state.

\section{Conclusions}

Respondents felt stigmatized and psychologically and morally traumatized. Isolation is a difficult experience and some negative emotions as expressed by previous studies were experienced by the respondents. There is need for increased testing capacity, timely results, early evacuation and creation of more isolation centres in Lagos State due to the rising number of cases and shortage of bed space. 


\section{Background}

The World Health Organization (WHO) in January 2020 declared the outbreak of the novel coronavirus disease (COVID-19) caused by SARS-CoV2 a Public Health Emergency of International Concern(1). It was subsequently declared a pandemic in March 2020. Despite efforts by WHO and public health authorities around the world to contain the COVID-19 outbreak(1). China was the first country affected by the pandemic of COVID-19, caused by SARS-CoV2 (1). Several unique characteristics of China's COVID-19 epidemic patterns and its management policy prompted a heightened public mental health crisis(2). Globally, report from Worldometer on the 27th of May revealed that the virus had spread in 212 countries around the world with a total of 5,709,551 confirmed cases with 2,451,226 recoveries and 352,753 deaths(3).

Africa is faced with increased crisis and concern in the public health sector as cases of COVID-19 rise daily. As at 27th of May 2020, the continent had 119,454 cases, 48,607 recoveries and 3,592 deaths(4), indicating the need to rise to the challenges that the pandemic is placing across the continent. The Nigeria Centre for Disease Control (NCDC) announced the first confirmed case of the pandemic of COVID19 in Nigeria on 27th February 2020. By the 27th of May 2020, Nigeria had a total of 7,526 confirmed cases, 2174 recoveries and 221 deaths(5) with Lagos State as the epicentre of the pandemic in the country. The state had at least 6 isolation centres as of 16th May 2020.

Quarantine and isolation have been used as measures to curtail the spread of COVID-19. Quarantine is the separation and restriction of movement of people exposed to a contagious disease in order to determine their status, hence, reducing the risk of infecting others(6) while isolation is the separation of people diagnosed with a contagious disease from people who are not sick. The two terms are used interchangeably especially in communication with the public(7). Some of the negative impact of quarantine are psychological effects such as post-traumatic stress symptoms, confusion, and anger. Stressors for longer duration of quarantine include infection fears, frustration, boredom, inadequate supplies, inadequate information, financial loss, and stigma with long lasting effects suggested by some researchers(8).

Stigma is a global barrier to health-seeking behaviours(9) resulting in various forms of discrimination leading to reduced or lack of social acceptance or opportunities to individuals or group. Social stigma in the context of health is the negative association between a person or group of people who share certain characteristics of a specific disease(10). In an outbreak, this may mean people are labelled, stereotyped, discriminated against, treated separately, and/or experience loss of status because of a perceived link with a disease(10). The COVID-19 pandemic is causing a lot of stigmatization because the disease is new with so many unknown factors, this induces fear in people resulting in their negative reaction towards the disease(10). The current COVID-19 outbreak has provoked social stigma and discriminatory behaviours against people perceived as suspect or confirmed living with the virus. Everyone is at risk due to community transmission and frontline health care workers are not exempted. This study therefore 
aimed to report the experiences of frontline health care workers treated for COVID-19 infection in Lagos State Nigeria.

\section{Methods}

\section{The aim, design and setting of the study}

We conducted a qualitative study on frontline health workers receiving treatment for COVID-19 at two isolation centres in Lagos, and one that was self-isolating due to lack of space at the isolation centres. This was done to have a broad range of experiences depending on where an individual was isolated. The study was also conducted to explore the issue of stigmatization based on the experiences of the frontline health care workers recovering from COVID-19 infection.

The inclusion criteria were frontline health workers recovering from COVID-19 and were engaged in the hospital or on the field during the outbreak. We purposively sampled the participants in this study. The exclusion criteria were inability to participate in two or more interviews by an individual during the study period. The interview was conducted within a period of eleven days (5th May to 15th of May, 2020) with a total of twelve participants engaged in the study. We conducted face-to-face in-depth interviews of 5 medical doctors (2 Medical epidemiologists), 1 veterinary epidemiologist, 1 laboratory epidemiologist, 2 nurses, 1 pharmacy technician and 2 hospital staff hygienists. The participant that was self-isolating was interviewed over the phone. The interview method was adapted for greater privacy and confidentiality in exploring individual views and in-depth information. This method is also more suitable for sensitive issues like living with COVID-19.

Four Epidemiologists interviewers were involved in data collection. We determined the number of respondents by interviewing frontline health workers that met the inclusion criteria until 'saturation point' was reached where no new information was generated. The respondents' comments were transcribed verbatim by the interviewers to effectively communicate the experiences of the respondents.

Frontline health workers are people that provide direct services to their communities. Providing lifesaving care and treatment, and investments in their training and ongoing support leading to tremendous health and economic returns. Frontline health workers consist of various health workers such as nurses, midwives, community health workers, doctors, pharmacists and others that provide care directly to their communities(11).

\section{Checklist}

A checklist was developed for this study and face validity was done by a team of field epidemiologists. This was to guide the interviewers during the discussion. This included questions like: What do you understand by COVID-19? What are the ways COVID-19 could spread? Any idea on how you got exposed to the virus? How did you feel when you were classified as confirmed case? In what way did your colleagues react to your being positive? Did you observe any form of stigmatization by your community? 
How have you been coping? What are your challenges so far regarding your mental health and isolation? What are the measures that can be taken to alleviate what you are passing through? Were you able to disclose your status to your family and friends? If yes, what was their reaction? If no, what were your reasons for not disclosing your status to your family/friends? What are your recommendations to people that are living with COVID-19? Any recommendation to improve on services rendered so far? Probing questions, such as "Please tell me more about that", was used to enhance the depth of discussion.

\section{Data analysis}

We conducted qualitative analysis of our data using the Colaizzi's phenomenological method. Descriptive phenomenology reveals the "essence" or "essential structure" of a phenomenon under investigation. We collected data using explicit first person accounts experience from face-to-face interviews and online interview(12). We conducted rigorous analyses using the seven vital steps while sticking to the data; familiarizing ourselves with the data, identifying significant statements with direct relevance to the phenomenon under investigation, formulating meanings, clustering themes, developing exhaustive description of the phenomenon while incorporating all the themes produced, producing the fundamental structure and seeking verification of the fundamental structure by some of the participants. This method of qualitative analyses is commonly used in the health sciences(12).

\section{Results}

The age range of those interviewed was 27 to 45 years of age, out of which seven were females and five males.

Table 1: Characteristic of respondents 


\begin{tabular}{|llll|}
\hline s/no & age & sex & occupation \\
\hline 1 & 42 & Female & Epidemiologist \\
\hline 2 & 42 & Male & Epidemiologist \\
\hline 3 & 43 & Male & Epidemiologist \\
\hline 4 & 45 & Male & Epidemiologist \\
\hline 9 & 33 & Female & Epidemiologist \\
\hline 5 & 29 & Female & Medical Doctor \\
\hline 5 & 34 & Male & Medical Doctor \\
\hline 7 & 27 & Female & Nurse \\
\hline 8 & 35 & Female & Nurse \\
\hline 10 & 33 & Female & Pharmacy technician \\
\hline 11 & 45 & Male & Hygienist \\
\hline 12 & 40 & Female & Hygienist \\
\hline
\end{tabular}

Theme: Knowledge on COVID-19 by respondents

All the respondents $(n=12)$ had knowledge of the virus while two $(n=2)$ of the 12 respondents stated that transmission could be air-borne due the high rate of infectivity of the virus. Their knowledge of COVID-19 was indicated under the subthemes; knowledge of the virus, mode of transmission and prevention/control.

\section{Theme: likely means of exposure}

Eleven respondents reported that they had primary contact with confirmed case or cases $(n=11)$ while one was a secondary contact of a confirmed case. All the participants $(n=12)$ likely place of exposure was at workplaces (hospitals/clinics) or at their various duties on the field during the outbreak investigation and mitigation. This was because a number of their colleagues tested positive and they had contacts with them before they went for testing. A respondent was not sure if the infection was acquired at the place of work.

\section{Theme: Reactions}

- Reactions form positive patients $(n=12)$

Reaction of positive frontline health workers on receiving the news includes initial denial due to being asymptomatic $(n=2)$, feeling bad $(n=3)$, anxiety, crying; some due to the fear of stigmatization, weakness, 
distress, pain, feeling disoriented, none specific reaction because the patients mind was prepared by a superior officer, looking forward for evacuation due to stigmatization by family members.

Other positive reactions despite tested positive were faith in God, bracing up to face the worst outcome after taking the test, overcoming the shock phase before the result was out, consoled by the recovery rate despite the test outcome, positivity that they will be negative in a few days, some felt that the situation could be avoided if only they wore mask while attending meetings and the issue of death did not cross their mind at any point.

\section{- Reaction from colleagues}

Majority of the respondents' colleagues $(n=7)$ were empathic, supportive, always called and encouraged the patients to stay positive, prayed fervently for the patients, assured patients that they will come out victorious and came visiting with provisions. Emails were sent by the Head of the organization to encourage those that got infected in the line of duty and to reassure them of continued support and also wished them quick recovery. Some immediate supervisors called to encourage and counsel patients. A patient had a consultant psychiatrist as a supervisor who helped the patient psychologically and also engaged his psychiatrists' colleague in the counselling process. A respondent's team lead immediately started crying when informed of the patient's positive status. Both the team and pillar leads were highly supportive as stated by some respondents.

On the contrary, the experience of others, $(n=3)$ of the respondents had negative reactions from their colleagues, they were stigmatized by their colleagues. Reactions includes colleagues distancing themselves, accused of bringing the infection to the health facility, was told by colleagues they promised never to eat food procured by her at the health facility.

two respondents $(n=2)$ had no reaction from colleagues because they were at home when their results came out.

- Reaction of the family and friends

Six $(n=6)$ of the respondents disclosed to their family and friends about their disease status while the rest did not disclose their status. Two of the respondents that disclosed their status had negative response from family members while the rest $(n=4)$ revealed that they got maximum support from their family and friends which turned out to be helpful emotionally and psychologically. They were prayed for, encouraged, and visited by family members and friends.

The rest of the respondents $(n=6)$ kept their status away from their family members for fear of inflicting distress, worries and possible psychological breakdown.

- Reaction by community 
The community was oblivious of the fact that these patients were positive $(n=12)$, partly because some of them were evacuated from the hospitals where they worked and those staying in the hotels informed the authorities of their status for their rooms and the environment to be decontaminated after departure to the isolation Centre.

\section{Theme: Challenges and coping methods}

Challenges mentioned were late processing of samples, delay in getting results up to two weeks after the test was done and late evacuation to the isolation centre. All the respondents $(n=12)$ related the challenges they went through during the period of isolation. While at the isolation centre, patients struggled with loneliness, insomnia, anxiety, movement restriction, worries about the welfare of their families at home, long duration of stay at the isolation centre about two weeks and yet to be discharged, lack of empathy by some of the staff at the isolation centre, the psychosocial group counselling made some patients more anxious instead of receiving comfort. Adverse side effects of drugs administered $(n=3)$; some adapted to it after some days while others could not. Keeping their status secret from family members because they didn't want them to be worried was challenging to some. Other challenges include stigmatization by the health workers at the centre; the workers seemed to be distancing themselves from the patients while wearing complete PPE. Not receiving the medical care that they expected, being shouted at for mistakenly shutting the door, faulty doors and windows in a patients bath room and the timing of the meals was not also acceptable to some (provision of meals at late hours).

Some of the coping mechanism employed were phone calls, surfing the web, listening to music, reading spiritual books, watching movies, working on some initial projects, attending webinars and working from the isolation centre.

\section{Theme: Recommendations}

- Recommendation for positive patients

Recommendations made by $(n=12)$ of the respondents during the period of isolation include: they should put their trust and faith in God; positives should be optimistic staying in isolation centre is not forever; and they should not think of stigmatization but to survive first. Others were: they should engage in activities that make them happy and shouldn't feel guilty for being positive and "COVID-19 is not a death sentence".

- Recommendations to improve the outbreak response and case management

To improve the outbreak response and case management, recommendations were made by all the respondents $(n=12)$. Implementation of standard guidelines for procedures for people receiving treatment at the isolation centres. The guidelines should be well communicated to the persons being brought for admission. There should be a single treatment policy in all the isolation centres. Increase testing capacity and prompt processing of samples collected so that results can come out in good time. Results of 
samples collected should be communicated not more than 48 hours after collection. A respondent suggested that the number of patients per tent should be reduced due to overcrowding.

Creation of more public awareness about the disease. The staff in these centers should be more empathetic to patients. Stigmatization by isolation centre staff should be reduced as much as possible. Training and retraining of isolation centres staff (case management team and hygienists) on how to interact with people recovering from COVID-19.

Timely evacuation of positive persons and the use of ambulance should be discouraged for both evacuation and home return after treatment to avoid community stigmatization. Equipping of Health facilities to tackle the disease. There is a need for a counsellor to be made available at the isolation centre to screen and identify those who need psychological support and provide such. Regularization of meal timetable at the isolation centres.

Table 2: Quotations of respondents under the various themes and subthemes 


\section{$\begin{array}{lll}\text { Theme } & \text { Subthemes } & \text { Quotations }\end{array}$}

Knowledge of the virus
Knowledge on COVID-19 by respondents
Mode of transmission
- "Spread through the crowd without social distance"

- $\quad$ "A viral infection transmitted via aerosol, droplets or contaminated surfaces. By touching the face with contaminated hand (nose). I am not too sure if food can transmit it."

- "It is spread through droplets and aerosol when an infected person, speaks, cough or sneezes. Can be transmitted through contaminate hands or environment."

- "COVID-19 is a viral disease that is transmitted through droplets from the mouth, and nose.

Transmission can occur through direct contact of droplets with the eyes, nose and mouth or indirectly by touching surfaces contaminated with droplets. The hand serves as a means by which the droplets are carried to from contaminated surfaces to the mouth, nose and eyes."

- "caused by virus droplet, I feel it is airborne due to the high rate of infectivity. I feel many people have it though they may be asymptomatic like me"

- $\quad$ "Viral infection, transmitted through droplets from coughing, sneezing and touching surfaces"

- $\quad$ "Is a viral disease caused by SARS-CoV-2 that is highly infectious, spread from person-to-person through droplets from respiratory tracts of infected persons to their close contacts when they cough, sneeze or shout and can also be transmitted when someone comes in 
contact with contaminated surfaces and/or fomites and touches his nose, eyes or mouth. It can also be transmitted via aerosols but airborne transmission has not been reported."

Prevention/control

"Preventive measures include Social distancing (2 meters), use of hand sanitizers ( $70 \%$ alcohol) to disinfect the hand, proper washing of the hand under running water for about 30 seconds, cleaning of high touched surfaces with disinfectant, the use of face mask and staying away from high risk areas."

- $\quad$ "The spread can be prevented or reduced by ensuring strict environmental hygiene, regular washing of hands with soap and running water for at least 20 seconds or the use of hand sanitizers with at least $60 \%$ alcohol, cough into flexed elbow or a tissue and immediately disposed hygienically, keep at least 2 meters' physical distance and avoid crowded environment and the use of face mask."

- "Keeping social distance at two meters or six feet and the use of face mask "

- "Exposed at the Primary Health Centre where I work"

- $\quad$ "Exposure from an outpatient in the hospital where I worked"

- "I can't really tell but I came in contact with a staff of the hospital that later on became positive, I had no symptoms, I went and tested because my contact became positive"

\section{Likely means of exposure}

- "Colleagues at the hospital, some of them tested positive"

- $\quad$ "Place of work, I was engaged in case investigation and had a lot of contact with positive and suspected cases"

- "Not really sure but feels I feel it's from my place of work"

- "We managed a positive patient at work. I wasn't the primary person that managed but one of my colleagues."

- "I must have been exposed through infected friends at my place of work. Some of my colleagues were infected and as contacts I went for my test and it was positive."

- "I think I was exposed at my place of work. Some of my close colleagues tested positive to the disease warranting me to also check my status and it turned out positive."

- "I had contact with few confirmed cases and a lot of suspected cases in the line of duty even though I was observing the safety measures." 
Immediate reactions form positive patients
- "I really don't know, I am a frontline health worker and in the case investigation team and my work involves filling of case investigation forms for suspect cases, so I wouldn't know if some are positive cases"

- $\quad$ "The infection was contracted through colleagues at the treatment centre and my screening test came out positive"

- "I was already looking forward for evacuation because it was delayed"

- "Initial denial because I don't have any symptom"

- "Weakness, comforted myself on finding out that I was not the only positive case in the facility and putting my faith in God"

- $\quad$ "Denied, because I had no symptoms, I was anxious and I cried"

- "Accepted it when I was told by my resident advisor, self-denial because I was asymptomatic, I wanted to be tested again to be sure but that was not accepted"

- $\quad$ "I am a strong believer in the supreme being, so I left everything to faith"

- $\quad$ "Distress, pain because I have been on admission for over a week at Saint Nicolas hospital before I tested positive.

"I felt bad but I was consoled that the recovery rate is high. I also had it before my grand mum. I felt I infected her and I felt very bad about that. I cried the first few days. Death did not cross my mind at any point."

- $\quad$ "I did not feel bad I knew that I would be negative within a few days to week. Though, I felt I could have avoided being positive. This would have been accomplished by using face mask while attending meetings. I was not symptomatic, and I saw that as a good prognosis."

- "I had encouraged myself and brace up for whatever outcome. So when I was called that it was positive, I had already gone through that phase of shock, I took it in good fate and looked forward to be evacuated"

- $\quad$ "Though felt bad initially because I thought I had been careful enough not to get infected but knew it's just a matter of time that I will be let out of the treatment centre"

- $\quad$ "Because of the delay in results and the symptoms I already had, I was already expecting to be positive."

"I was distraught, I didn't feel happy about it"

"Telling my family helped me to settle down by 
Reactions of their families and friends talking to my wife regularly and being encouraged by my wife"

- " "I stay with my uncle and his family, their immediate reaction was dramatic, I did expect the reaction but I was not ready for it. I was happy coming to the isolation centre because of the reaction of my family members"

- "My family helped by showing me care, they kept calling, encouraging me and telling me about remedies that might be of help. In some instance I say no that the measures are extreme."

- $\quad$ "Disclosed it to my sister in-law and my friend. They were really empathic and supported me throughout my period of stay"

- "My husband has been very supportive though I did not inform my children so that they will not be worried."

- "I live with my parents, my mom felt awfully bad, my dad felt bad, but he denied that I can't be positive"

- "I could not inform my immediate family members; my wife will be devastated. I want to prevent the trauma. She will become anxious to see me and it will be difficult for her to travel to Lagos"

- "I didn't inform members of my family for fear of their psychological breakdown"

- $\quad$ "I could not inform my wife till now for fear of her not taking the news well. Informed my brother who is a medic too and he has been supportive"

- "Family? No, friends? Yes"

- $\quad$ “No, I didn't inform them because I felt it was unnecessary especially for the fact that I was not symptomatic and I know that I will get over it"

"Stigmatized by colleagues, a colleague of mine said that I was the one that brought the disease to them, that I use to bring some Hausa people into the health facility."

- "Distancing themselves from me"

- "No negative reaction from colleagues because I was already at home when my results came out"

- "None"

- "My colleagues were empathic and always called to encourage me"

- Some of my colleagues reacted in not too good manner and one of them promised never to eat food procured by me again."

Reaction from colleagues
- "Supportive"

Page $13 / 22$ 
"Most of my colleagues were supportive, they came visiting and came with gifts. Only a few behaved as if it could not have been them. My immediate supervisor called to encourage and counselled me. $\mathrm{He}$ is a consultant Psychiatrist; he did a good job on me. He also shared my contact with another Psychiatrist who called and encouraged me as well."

- "They were quite supportive. They called frequently, prayed fervently for me, ensured me that I will come out victorious and some came visiting with goods. My team lead immediately started crying when, I informed her of my positive status. Both my team and Pillar leads were highly supportive."

- "They were supportive and tried to calm me down. I also received calls from my colleagues outside Lagos."

"Had very good support from colleagues but clinical and non-clinical which was encouraging enough. Calls and visits regularly was strengthening. Non-specific untoward reaction was noticed. Senior colleagues who had the same experience called to encourage me and also close friends who were not at my present location"

- $\quad$ "they were sympathetic and they shared in my fears and tried to allay my fears and told me that I am fine especially the fact that I am not symptomatic"

Community

Reaction
"Oblivious of the fact that she is positive"

- "Community not aware"

- "The community are not aware that I tested positive"

- "None"

- "When I informed the manager of the hotel where I stay, she prayed that I get well soon, I taught the manager how to decontaminate my room and she was thankful."

- "Not really"

- 'Living very far from home, couldn't tell my wife, I didn't want to create unnecessary worries."

- "stigmatization by the health workers here"

- "I cried because I might be stigmatized. Only temperature is checked here. Twice daily. The window of my room and the door of the bath room were not good."

- $\quad$ "Loneliness, worried about my children at home, staying because it is compulsory, late processing of my samples, my result came out about two weeks after taking the test and I have stayed for about 2 weeks already at the isolation centre" 
Challenges and copping methods
Challenges and copping methods
- "Some of the staff here are not empathetic, the psychosocial group counselling made me more anxious instead of calm me down"

- $\quad$ "The workers at the hotel seem to be distancing themselves from us even while wearing complete PPE while mopping my room, I was told to stay inside the bathroom which I did until the person finished the mopping; being shouted at because I mistakenly shut my door; away from home and alone"

- $\quad$ "Not been able to move freely, not been able to see my immediate family for some time."

- "Not been able to move freely as I desire, I could not do my usual work. However, I spent the time attending webinars and working from my isolation room."

- "Restriction of my movement, the effects of the drugs, meals were not coming at the right time, poor treatment and avoidance mechanism established by the case management team at the isolation centre"

- "The solation centre was not conducive, not enough space in the tent, didn't have enough sleep, noise from television from other patients. I copped through; watching movies, having long calls with family members, working on my laptop on some of the works that I started."

- $\quad$ "Not been able to move freely as I desire. A bit of worry when I noticed a symptom that was not present before (loss of smell)."

- "I am not finding the isolation easy, especially the fact that my result is still pending, there is no feedback from the laboratory and I have spent 16 days already in isolation waiting for my second result. have been watching movies in and out of sleep and I have been talking with family and friends"

- $\quad$ "No challenges personally, I coped through using my phone, listening to music and reading spiritual books,"
- "They should put their trust in God" for positive cases
- "They should surround themselves with things that make them happy, they shouldn't feel guilty for being positive"

- "Put their faith in God and stay positive"

- $\quad$ "Positives should be optimistic staying in isolation centre is not forever, they should not think of stigmatization but to survive first."

- "COVID-19 is not a death sentence"

"House to house testing, increase testing capacity and samples should be processed immediately so that 
Recommendations to improve the outbreak response and case management results can come out in good time."

- "Create more public awareness about the disease"

- $\quad$ "The staff in this centre should be more empathetic to patients, there should be standard guidelines for procedures, timely processing of samples collected, and timely dissemination of results, timely evacuation of positive persons."

- $\quad$ "The process of evacuation of patients should be considered because of community stigmatization."

- "Showing of more care by the health workers"

"People should be alerted about their status on time and not being worried for several days or weeks before result notification. Stigmatization by isolation centre staff should be reduced as much as possible. Health facilities should be equipped more to tackle the disease. The government should make house to house screening of population mandatory."

- $\quad$ "There should be plan for discharge after admission here, the use of ambulance should be discouraged for both evacuation and return home after treatment. People should be alerted about their status on time and not been worried for several days of weeks before result notification. Decontamination of the items owned by the persons discharged should be done in their rooms and not outside."

- $\quad$ "There is a need for a counsellor at the isolation centre. The person will screen and identify those who need psychological support and provide such. Training and retraining of isolation centre staff on how to handle cases is important."

- $\quad$ "Regularize mealtime. Case management team should show more care to patients. Results of samples collected should be communicated not more than 48 hours after collection. There should be a single treatment policy in all the isolation centers

- $\quad$ "Special treatment for all the frontline health workers. Reduce the number of patients per tent."

- $\quad$ "Support the government and Nigeria Centre for Disease Control by adhering strictly to all the preventive measures to avoid transmission."

- "Coordination; the whole response is not well coordinated and there is need for timely laboratory feedback. Whether you are a frontline health worker or not, the delay was a general case"

\section{Discussion}


The respondents were quite knowledgeable about COVID-19 which could be associated with their professional background and being COVID-19 frontline health care workers. They were at high risk of acquiring the infection based on the nature of their work. This study found out that initial reaction to the news of testing positive to COVID-19 was denial, anxiety, fear, distress, disorientation, worries and challenges such as stigmatization, movement restriction, insomnia, loss of smell (anosmia), unconducive environment similar to other qualitative studies(13-18). Qualitative studies also identified a range of other psychological responses to quarantine, such as confusion, numbness, fear, grief and anxietyinduced insomnia(15,19-21). It is understandable that there is confusion, anxiety, and fear among the public due to the increasing incidence and mortality associated with COVID-19. Unfortunately, these factors are also fueling harmful stereotypes. This can undermine social cohesion resulting in social isolation of groups as a result of stigma (10).

The impact of stigma results in difficulty in more severe health problems and difficulty in handling the disease outbreak. Stigma can drive people to hide the illness to avoid discrimination, prevent people from seeking health care immediately and discourage them from adopting healthy behaviours $(10,20)$. An environment needs to be created in which the disease and its impact can be discussed and addressed openly, honestly and effectively. Governments, citizens, media, key influencers and communities have an important role to play in preventing and stopping stigma surrounding people. We all need to be intentional and thoughtful when communicating on social media and other communication platforms, showing supportive behaviors around the new coronavirus disease (COVID-19)(10).

Quarantine is usually unpleasant and involves loss of liberty, physical separation from loved ones, uncertainty of disease status, boredom and may result in dramatic effects such as suicide(22). A study of quarantined (9 days) hospital staff who might have come into contact with SARS reports that quarantine results is associated with symptoms of acute stress disorder and are more prone to exhaustion, detachment from others, anxiety when dealing with febrile patients, irritability, insomnia, poor concentration and indecisiveness, deteriorating work performance, and reluctance to work or consideration of resignation(23). A study on hospital staff examined 3 years after quarantine indicated that $9 \%$ (48 of 549) of the sample had high depressive symptoms and about $60 \%$ ( 29 of 48 ) were previously quarantined while only $15 \%$ (63 of 424) of the group with low depressive symptoms had been quarantined indicating the dangers associated with quarantine(24).

This study revealed a respondent complied to the isolation because it was compulsory. Individuals quarantined/isolated need to understand the situation through the means of effective communication. Majority of severe effects of quarantine/isolation result from imposition of restriction of freedom. There is less distress and long-term complications with voluntary isolation. Public health officials should stress the importance of voluntary self-isolation and the duration of isolation practiced should be short and should not be altered except in extreme circumstances $(8,25)$.

Mitigation of stigma for COVID-19 infection should focus on enacted stigma (acts of discrimination and mistreatment), felt-normative stigma (demeaning community norms and values), internalized stigma (the 
ways that persons accept negative perspectives toward a group(s) they may belong to) and anticipated stigma (concerns that one will experience future discrimination and bias)(26) for holistic approach in tackling of stigma in the current outbreak.

Recommendations made to people that tested positive to COVID-19 were to put their faith in God, stay optimistic, think of themselves first irrespective of stigmatization and be conscious of the fact that testing positive to COVID-19 is not a death sentence. This can be achieved through proper counselling and educating of people recovering from COVID-19 at the various isolation centres.

Other recommendations were implementing standard guidelines for people at the isolation centres. Increase testing capacity, prompt processing of samples and communication of results within 48 hours of sample collection. This is necessary to eliminate long waiting and long period of anxiety while waiting for the results. This measure will also limit the spread of the virus by those people that tested positive. This is in line with the need of immediate evacuation of people that tested positive to COVID-19. Delay in the evacuation is also a means of further spread of the disease especially for patients that can't selfisolate due to lack of enough rooms in their houses.

There is need for health care staff at the isolation centres to be empathic with people recovering from COVI9-19 which can help in the psychological healing process. The effect of isolation especially when imposed on a person results in psychological and moral trauma, coupled with the side effects of the drug which some of them had to manage. Hence, the respondents advocated for training and retraining of staff at the isolation centres on how to interact with people recovering from COVID-19 at the isolation centres to improve on the current situations. The need for physically present counsellor to improve the psychological and emotional well-being of people that tested positive at the various isolation centres who are in need of such services at the centres was also advocated for. A respondent complained about unconducive isolation tent, too many people and noise from television resulting in his inability to rest well. There is a need for the management to address the issue of overcrowding. Individuals that tested positive to COVID-19 and can self-isolate at home should be allowed to do so since there is consistent increase in the number of cases in Lagos State as the epicentre of the disease there is mounting pressure on bed space for isolation. This will alleviate the problem of bed space in the face of the pandemic. The study has also revealed the need for the creation of more isolation centres in the state due to the rising demand.

\section{Conclusions}

The study has revealed stigmatization, psychological and emotional trauma frontline health workers experienced while isolating during their recovery period from COVID-19. Their reactions were similar with other qualitative studies. Recommendations were also made by respondents to improve on the current existing system on outbreak investigation and management of people recovering from COVID-19 infection at the isolation centres. It is hoped that these recommendations will be acted upon by the relevant authorities for better output in the face of the pandemic. 


\section{Abbreviations}

Not applicable

\section{Declarations}

\section{Ethics approval and consent to participate}

This study was reviewed and approved by the Institutional Review Board of the Nigerian Institute of Medical Research (Project N0: IRB/20/048). Verbal informed consent was gotten from all participants before they were interviewed. The participants were assured that their response will be anonymous and they have nothing to fear by revealing their thoughts.

\section{Consent for publication}

All participants were informed and gave verbal consent for the publishing of this study.

\section{Availability of data and materials}

This study is a qualitative study and all the contributions of the respondents was recorded verbatim and tabulated as part of the results obtained. There is no outstanding materials or data pertaining this study to be submitted.

\section{Competing interests}

The authors declare that there is no competing interest.

\section{Funding}

Not applicable

\section{Authors' contributions}

AVK wrote the concept note, was involved in data collection, data analyses and prepared the manuscript draft. OSI prepared the proposal and got ethical approval and was involved in data collection and analyses. POA partipated in data collection. JOO was part of the data collection. RU responsible for research concept. All the authors provided critical feedback on the framework and the manuscript draft. MSB did critical editing of final manuscript draft for intellectual content. All authors read and approved the final manuscript.

\section{Acknowledgements}


We appreciate the management at the isolation centres for allowing us to conduct the study and all the respondents that made this study possible by giving their consent and participating in the study. We acknowledge the Nigeria Centre for Disease Control (NCDC), Nigeria Field Epidemiology and Laboratory Training Programme (NFELTP) and Africa Field Epidemiology Network (AFENET).

\section{References}

1. WHO. Mental health and psychosocial considerations during the COVID-19 outbreak. 2020 [cited 2020 May 27]; Available from: https://www.who.int/docs/default-source/coronaviruse/mentalhealth-considerations.pdf?sfvrsn=6d3578af_2

2. Li H, Chen X, Huang $H$. The novel coronavirus outbreak: what can be learned from China in public reporting? 2020;9:5-7.

3. Wang W, Tang J, Wei F. Updated understanding of the outbreak of 2019 novel coronavirus (2019nCoV) in Wuhan, China. J Med Virol [Internet]. 2020 Apr 12 [cited 2020 May 27];92(4):441-7. Available from: https://onlinelibrary.wiley.com/doi/abs/10.1002/jmv.25689

4. Coronavirus in Africa: 119,454 cases; 3,592 deaths; 48,607 recoveries | Africanews [Internet]. [cited 2020 May 27]. Available from: https://www.africanews.com/2020/05/27/coronavirus-in-africabreakdown-of-infected-virus-free-countries/

5. Nigeria Coronavirus: 8,344 Cases and 249 Deaths - Worldometer [Internet]. [cited 2020 May 27]. Available from: https://www.worldometers.info/coronavirus/country/nigeria/

6. Centers for Disease Control and Prevention. Quarantine and isolation [Internet]. 2017. Available from: https://www.cdc.gov/quarantine/index.html

7. Manuell M-E, Cukor J. Mother Nature versus human nature: public compliance with evacuation and quarantine. Disasters [Internet]. 2011 Apr [cited 2020 May 27];35(2):417-42. Available from: http://www.ncbi.nlm.nih.gov/pubmed/21073672

8. Brooks SK, Webster RK, Smith LE, Woodland L, Wessely S, Greenberg N, et al. Rapid Review The psychological impact of quarantine and how to reduce it: rapid review of the evidence. Lancet [Internet]. 2020;395(10227):912-20. Available from: http://dx.doi.org/10.1016/S01406736(20)30460-8

9. Scott N, Crane M, Lafontaine M, Seale H, Currow D. Stigma as a barrier to diagnosis of lung cancer: patient and general practitioner perspectives. Prim Health Care Res Dev [Internet]. 2015 Nov [cited 2020 May 27];16(6):618-22. Available from: http://www.ncbi.nlm.nih.gov/pubmed/25660904

10. A guide to preventing and addressing social stigma associated with COVID-19 [Internet]. [cited 2020 May 27]. Available from: https://www.who.int/who-documents-detail/a-guide-to-preventing-andaddressing-social-stigma-associated-with-covid-19

11. Frontline Health Workers | Frontline Health Workers Coalition [Internet]. [cited 2020 May 27]. Available from: https://www.frontlinehealthworkers.org/frontline-health-workers 
12. Morrow, R., Rodriguez, A. and King N. Colaizzi's descriptive phenomenological method. Psychologist. 2015;28(8):643-4.

13. Yoon M-K, Kim S-Y, Ko H-S, Lee M-S. System effectiveness of detection, brief intervention and refer to treatment for the people with post-traumatic emotional distress by MERS: a case report of community-based proactive intervention in South Korea. Int J Ment Health Syst [Internet]. 2016 [cited 2020 May 27];10:51. Available from: http://www.ncbi.nlm.nih.gov/pubmed/27504141

14. Hawryluck L, Gold WL, Robinson S, Pogorski S, Galea S, Styra R. SARS control and psychological effects of quarantine, Toronto, Canada. Emerg Infect Dis [Internet]. 2004 Jul [cited 2020 May 27];10(7):1206-12. Available from: http://www.ncbi.nlm.nih.gov/pubmed/15324539

15. DiGiovanni C, Conley J, Chiu D, Zaborski J. Factors influencing compliance with quarantine in Toronto during the 2003 SARS outbreak. Biosecur Bioterror [Internet]. 2004 [cited 2020 May 27];2(4):265-72. Available from: http://www.ncbi.nlm.nih.gov/pubmed/15650436

16. Lee S, Chan LYY, Chau AMY, Kwok KPS, Kleinman A. The experience of SARS-related stigma at Amoy Gardens. Soc Sci Med [Internet]. 2005 Nov [cited 2020 May 27];61(9):2038-46. Available from: http://www.ncbi.nlm.nih.gov/pubmed/15913861

17. Reynolds DL, Garay JR, Deamond SL, Moran MK, Gold W, Styra R. Understanding, compliance and psychological impact of the SARS quarantine experience. Epidemiol Infect [Internet]. $2008 \mathrm{Jul}$ [cited 2020 May 27];136(7):997-1007. Available from: http://www.ncbi.nlm.nih.gov/pubmed/17662167

18. Marjanovic Z, Greenglass ER CS. The relevance of psychosocial variables and working conditions in predicting nurses' coping strategies during the SARS crisis: an online questionnaire survey. Int J Nurs Studv. 2007:44:991-8.

19. Pan PJD, Chang S-H YY-Y. A support group for homequarantined college students exposed to SARS: learning from practice. J Spec Gr Work. 2005;30:363-74.

20. Pellecchia U, Crestani R, Decroo T, Van den Bergh R, Al-Kourdi Y. Social Consequences of Ebola Containment Measures in Liberia. Braunstein LA, editor. PLoS One [Internet]. 2015 Dec 9 [cited 2020 May 27];10(12):e0143036. Available from: https://dx.plos.org/10.1371/journal.pone.0143036

21. Wang $Y, X u B, Z$ hao $G$, Cao R, He X FS. Is quarantine related to immediate negative psychological consequences during the 2009 H1N1 epidemic. Gen Hosp Psychiatry. 2011;33:75-77.

22. Barbisch $D$, Koenig KL SF. Is there a case for quarantine? Perspectives from SARS to Ebola. Disaster Med Public Heal Prep. 2015;9:547-53.

23. Bai Y, Lin C-C, Lin C-Y, Chen J-Y, Chue C-M, Chou P. Survey of stress reactions among health care workers involved with the SARS outbreak. Psychiatr Serv [Internet]. 2004 Sep [cited 2020 May 27];55(9):1055-7. Available from: http://www.ncbi.nlm.nih.gov/pubmed/15345768

24. Liu X, Kakade M, Fuller CJ, Fan B, Fang Y, Kong J, et al. Depression after exposure to stressful events: lessons learned from the severe acute respiratory syndrome epidemic. Compr Psychiatry [Internet]. 2012 Jan [cited 2020 May 27];53(1):15-23. Available from: http://www.ncbi.nlm.nih.gov/pubmed/21489421 
25. Martinez-Alvarez $M$, Jarde A, Usuf E, Brotherton $H$, Bittaye $M$, Samateh AL, et al. COVID-19 pandemic in west Africa. Lancet Glob Heal [Internet]. 2020 [cited 2020 May 27];8(5):e631-2. Available from: http://www.ncbi.nlm.nih.gov/pubmed/32246918

26. Lancet T. COVID-19: learning from experience. Lancet. 2020;395(10229):1011.

\section{Supplementary Files}

This is a list of supplementary files associated with this preprint. Click to download.

- BHSRD2001905COREQguidelines.docx

- Checklist.docx 